\title{
Die Ostseestrategie der Europäischen Union: Entwicklung, Stand und Perspektiven eines neuen Ansatzes in der EU- Regionalpolitik
}

\author{
Stefan Gänzle und Kristine Kern*
}

\section{Makroregionale Strategien der Europäischen Union - eine Definition}

Makroregionale Strategien der Europäischen Union zielen auf Natur-, Funktions- beziehungsweise Kulturräume innerhalb Europas, die von einem Gebirgszug wie den Alpen, einem Binnenmeer wie der Ostsee oder auch einem Fluss wie der Donau maßgeblich geprägt werden. So verstand der damalige EU-Kommissar für Regionalpolitik, Paweł Samecki, eine Makroregion als ein aus unterschiedlichen Ländern und Regionen bestehendes Territorium, „das ein oder mehrere gemeinsame Charakteristika oder Herausforderungen“1 aufweist. Eine aktuelle Definition der Europäischen Kommission aus dem Jahre 2013 fasst makroregionale Strategien dementsprechend als Entwurf zur Bewältigung gemeinsamer Herausforderungen und als ,regionale Bausteine für eine EU-weite Politik, die nationale Ansätze in eine kohärentere Durchführung auf EU-Ebene einbinden."2 Makroregionale Strategien formulieren also einen Gesamtrahmen für transnationale Zusammenarbeit, der sich sowohl an Mitglied- wie auch Drittstaaten desselben geografischen Raumes wendet, und versuchen zugleich, einen Beitrag zur Umsetzung des in Artikel 174 Vertrag über die Europäische Union (EUV) primärrechtlich verankerten Prinzips territorialer Kohäsion zu leisten - in Ergänzung der bereits im Vertrag von Maastricht 1992 vereinbarten Ziele sozialer und wirtschaftlicher Kohäsion. Im Hinblick auf den EU-Finanzplan für den Zeitraum von 2014 bis 2020 waren die jeweils betroffenen Mitgliedstaaten von der Europäischen Kommission dazu angehalten, die Ziele der bereits bestehenden makroregionalen Strategien sowohl in ihren Partnerschaftsabkommen als auch den operativen Programmen zur Umsetzung der EU-Regional- und Strukturpolitik aufzugreifen. Im Kern bilden makroregionale Strategien nunmehr einen Referenzpunkt zur politikfeldübergreifenden Koordination von Akteuren auch über die Regionalpolitik im engeren Sinne hinaus. Dabei besteht die Absicht, Synergieeffekte sowohl durch eine bessere Verknüpfung von Instrumenten unterschiedlicher Politikbereiche über die Grenzen von Politiksektoren (Transport, Umwelt usw.) als auch Gouvernanz-Ebenen (regional-mitgliedstaatlich-europäisch) hinweg zu erzielen. Damit knüpfen makroregionale Strategien zwar an die bereits bestehen-

* Prof. Dr. Stefan Gänzle, Universitet i Agder, Kristiansand.

Prof. Dr. Kristine Kern, Universität Potsdam und Leibniz-Institut für Regionalentwicklung und Strukturplanung, Erkner.

Dieser Beitrag stellt eine Überarbeitung der Ergebnisse einer im Jahr 2013 verfassten Studie der Autoren dar: Kristine Kern/Stefan Gänzle: Towards Cruising Speed? Assessing the EU Strategy for the Baltic Sea Region, Swedish Institute for European Policy Studies, European Policy Analysis 17, Stockholm.

1 Paweł Samecki: Keynote Address on EU Baltic Sea Strategy Ministerial conference on the EU Baltic Sea Strategy, Stockholm, 18 September 2009, abrufbar unter: http://europa.eu/rapid/press-release_SPEECH-09-396_en. htm (letzter Zugriff: 1.10.2014).

2 Europäische Kommission: Bericht der Kommission an das Europäische Parlament, den Rat, den Europäischen Wirtschafts- und Sozialausschuss und den Ausschuss der Regionen zum Mehrwert der makroregionalen Strategien, COM (2013) 468, S. 4. 
den Euroregionen oder auch Europäischen Gruppierungen für territoriale Zusammenarbeit (EGTZ) an, betten aber die vergleichbaren Zielsetzungen in einen größeren territorial-naturräumlichen Rahmen, der sowohl Anreize zu funktionaler Kooperation als auch zur Identitätsstiftung bietet - darin besteht das grundsätzlich Neuartige bei diesem Ansatz der EU-Regionalpolitik.

Für alle bislang mit einer makroregionalen Strategie bedachten Räume der Europäischen Union gilt, dass sich die Europäische Union bereits im Vorfeld der Erweiterungsrunden in den Jahren 1995, 2004, 2007 und 2013 zu dem jeweils zentralen politischen Bezugspunkt darin entwickelt hat. Schon seit mehr als zehn Jahren ist die Ostsee ein nahezu vollständig von Mitgliedstaaten der Europäischen Union umgebenes EU-Binnenmeer. Neben den zwei Partnerstaaten Russland und Norwegen richtet sich die Strategie der Europäischen Union für den Ostseeraum (Ostseestrategie) denn auch maßgeblich an die acht EUStaaten - Dänemark, Deutschland (vertreten durch die norddeutschen Bundesländer Schleswig-Holstein, Hamburg und Mecklenburg-Vorpommern), Estland, Finnland, Lettland, Litauen, Polen und Schweden. ${ }^{3}$

Makroregionale Strategien sind in die europäische Mehrebenen-Organisation integriert, die auf unterschiedlichen Gouvernanz-Ebenen eine Vielzahl von Akteuren - Städte, Regionen und Mitgliedstaaten sowie auch zivilgesellschaftliche und wirtschaftliche Interessengruppen - aufweist. ${ }^{4}$ Primär zielen die Strategien darauf ab, die Koordination dieser Akteure im europäischen Mehrebenen-System vertikal (mit Blick auf die subnationale Ebene), horizontal (mit Blick auf die Mitgliedstaaten und Nachbarn) und politikfeldübergreifend (zum Beispiel Umweltschutz und Landwirtschaft) zu verbessern. Gleichzeitig stehen makroregionale Strategien dabei unter dem Vorbehalt der sogenannten, drei Nein ${ }^{6}$ : das heißt es sollen für ihre Umsetzung keine neuen Regeln, keine neuen Institutionen und keine zusätzlichen Finanzierungsmittel erforderlich sein. ${ }^{5}$ Vielmehr soll auf bereits bestehende rechtliche Grundlagen und Einrichtungen sowie europäische beziehungsweise nationale und regionale Fonds und Finanzierungsmöglichkeiten zurückgegriffen und durch eine verbesserte Koordination der an der Umsetzung beteiligten Akteure ein praktischer Mehrwert erzielt werden. Im Lichte der Finanz- und Wirtschaftskrise lassen sich makroregionale Strategien gerade auch deshalb als Instrumente dafür betrachten, zur wirtschaftlichen Entwicklung der jeweiligen Makroregionen beizutragen und auf diese Weise die Ziele der Europa 2020-Strategie und damit verbundener Programme wie beispielsweise Horizont 2020 in einem bestimmten geografischen Raum umzusetzen. Darüber hinaus sind makroregionale Strategien auch als Vehikel einer vertieften Europäisierung dieser Räume

3 Demgegenüber ist die Zusammensetzung der Mitglieder der Donaustrategie deutlich heterogener geblieben: unter den insgesamt 14 Staaten sind die neun EU-Mitglieder Bulgarien, Deutschland (nur Baden-Württemberg und Bayern), Kroatien, Österreich, Rumänien, Slowakei, Slowenien, Tschechien, Ungarn; die EU-Beitrittskandidaten Montenegro und Serbien; der sogenannte potenzielle Beitrittskandidat Bosnien-Herzegowina sowie vier Regionen der Ukraine und die Republik Moldau (Östliche Partnerschaft). Das von der Strategie der Europäischen Union für die Region Adria-Ionisches Meer betroffene geografische Gebiet umfasst vier Mitgliedstaaten (Griechenland, Italien - hier ausschließlich die Küstenregionen des Adriatischen und des Ionischen Meeres, Kroatien und Slowenien), ein Kandidatenland (Montenegro) und drei potenzielle Kandidatenländer (Albanien, Bosnien und Herzegowina und Serbien), die an den Heranführungsverfahren der Europäischen Union teilnehmen.

4 Vgl. Iveta Reinholde: How to Govern the Strategy? Whether to Govern at all ..., in: Žaneta Ozoliņa/Iveta Reinholde/Tom Rostoks (Hrsg.): The EU Strategy for the Baltic Sea Region: A Year After and Beyond, Riga 2010, S. 41-58, hier S. 51.

5 Vgl. Carsten Schymik: Modellversuch Makroregion. Die EU-Strategien für den Ostsee- und den Donauraum, Stiftung Wissenschaft und Politik: SWP-Studien 2011/S 01, S. 5-6. 
$\mathrm{zu}$ verstehen und lassen sich prinzipiell auch als ein „tool of European integration“ "6 beschreiben.

Nach einer kurzen Betrachtung der Entstehung der Ostseestrategie und ihres Gouvernanz-Aufbaus, verfolgt dieser Beitrag die folgenden Ziele: erstens die Arbeitsstruktur im Lichte der ,drei Nein' zu analysieren; zweitens die Wirkung auf bestehende Einrichtungen der regionalen Zusammenarbeit im Sinne einer politischen Mobilisierung und Koordinierung von Akteuren einzuschätzen; und drittens schließlich die Rolle von subnationalen, partnerstaatlichen und zivilgesellschaftlichen Akteuren bei der Implementierung der Strategie zu untersuchen. Abschließend werden die bei der Umsetzung der Ostseestrategie bislang erzielten Erfolge wie auch Unzulänglichkeiten insgesamt kritisch gewürdigt.

\section{Entstehung und Entwicklung der EU-Strategie für den Ostseeraum}

Scheinbar unaufhaltsam schreitet die „Makroregionalisierung“7 ${ }^{67}$ der Europäischen Union voran: Schon kurz nach der Verabschiedung der Strategien der Europäischen Union für den Ostseeraum im Jahre 2009 und für den Donauraum (Donaustrategie) im Jahre 2011 wurde die Europäische Union geradezu von einer Art ,makroregionalem Fieber ${ }^{6}$ erfasst. ${ }^{8}$ So forderte der Europäische Rat die Kommission im Dezember 2012 auf, auch für das Adriatisch-Ionische Becken eine Strategie auszuarbeiten und bis zum Ende des Jahres 2014 vorzulegen. Im Mai 2013 regte das Europäische Parlament in einer Resolution dazu an, auch den Alpenraum mit einer makroregionalen Strategie zu bedenken. Ebenso bestehen Überlegungen, ähnliche Strategien für den Nordseeraum, den westlichen Atlantikbogen und das westliche Mittelmeer zu entwickeln. ${ }^{9}$ Die litauische EU-Ratspräsidentschaft hatte in ihrem Programm für die zweite Jahreshälfte 2013 erstmals das Leitbild eines „Europe of macro-regions"10 entworfen und eine Reihe von Veranstaltungen - wie etwa auch das Jahresforum in Vilnius - dazu genutzt, diese Thematik zu einem festen Bestandteil der Agenda europäischer Politik zu machen. Im Hinblick auf die lettische Ratspräsidentschaft 2015 ist davon auszugehen, dass das Thema auch in Zukunft auf der politischen Tagesordnung der Europäischen Union stehen wird.

Im Unterschied zu anderen Makroregionen Europas beziehungsweise der Europäischen Union kann sich die Kooperation im Ostseeraum auf einen besonders reichen Erfahrungsschatz wirtschaftlicher, politischer und kultureller Zusammenarbeit und Transnationalität stützen. ${ }^{11}$ Damit nimmt sie gewiss eine Sonderstellung unter den Makroregionen Europas ein und stellt sicherlich ,eine neue Qualität makroregionaler Strategieentwicklung auf eu-

6 Alexandre Dubois/Sigrid Hedin/Peter Schmitt/José Sterling: EU Macro-regions and Macro-regional Strategies - A Scoping Study, Nordregio Electronic Working Paper 4, Stockholm 2010, S. 10.

7 Eckart D. Stratenschulte: Von der Ostsee in die Welt? - Schlussfolgerungen der Ostseestrategie für weitere makroregionale Strategien in Europa, in: Eckart D. Stratenschulte (Hrsg.): Das europäische Meer. Die Ostsee als Handlungsraum, Berlin 2011, S. 159-173, hier S. 163.

8 Stefanie Dühr: Baltic Sea, Danube and Macro-Regional Strategies - a Model for Transnational Cooperation in the EU?, Notre Europe Study \& Research 86, Paris 2010, S. 3.

9 Vgl. hierzu Stefan Gänzle/Kristine Kern (Hrsg.): A ,Macro-regional Europe“ in the Making? Theoretical Approaches and Empirical Evidence, London 2015 (in Vorbereitung).

10 Litauische Ratspräsidentschaft: Program of the Lithuanian Presidency of the Council of the European Union. July 1 to December 31, 2013, abrufbar unter: http://static.eu2013.1t/uploads/documents/Presidency_program me_EN.pdf (letzter Zugriff: 1.10.2014).

11 Vgl. Bernd Henningsen: Die Ostsee als Handlungs- und Kulturraum: Geschichte, Politik und Kultur, in: Eckart D. Stratenschulte (Hrsg.): Das europäische Meer. Die Ostsee als Handlungsraum, Berlin 2011, S. 9-20. 
ropäischer Ebene“"12 dar. So bildet beispielsweise die Tradition der mittelalterlichen Hanse bis heute einen unter den Ostseeanrainerstaaten zumeist positiv besetzten Bezugspunkt. Es war auch gerade der Ostseeraum, der bereits zu Zeiten des Ost-West-Konfliktes in den 1970er Jahren die Bühne für die ersten blockübergreifenden Formen zwischenstaatlicher Kooperation bereitstellte, wofür zutreffend der Begriff der ,antagonistischen Kooperation“13 geprägt wurde. Hierzu gehören beispielsweise die im Rahmen der Helsinki-Konvention zwischenstaatlich vereinbarten Schritte zur Begrenzung beziehungsweise schrittweisen Verringerung der Meeresverschmutzung oder auch Städtepartnerschaften - wie etwa die seit 1953 zwischen Turku und Leningrad (St. Petersburg) bestehende Kooperation.

Seit den 1990er Jahren entwickelte sich schließlich die Europäische Union immer mehr zum zentralen Akteur innerhalb des Ostseeraumes. So legte bereits im Oktober 1994 die Europäische Kommission einen ,Ansatz der Union gegenüber dem Ostseeraum“ (Union Approach towards the Baltic Sea Region) vor. Nach der EU-Osterweiterung bereitete die in der Baltic-Europe Intergroup organisierte Gruppe von ,Ostseeparlamentariern “ um Christopher Beazley, Alexander Stubb und Toomas Hendrik Ilves im Jahr 2005 eine Strategie vor, deren Ziel sein sollte, das Potenzial der nunmehr innerhalb der Europäischen Union vereinten Ostseeregion besser zu nutzen. ${ }^{14}$ Im Kern war der an den Kommissionspräsidenten José Manuel Barroso gerichtete Strategieentwurf der Parlamentarier vor allem auf die Außenbeziehungen gerichtet und verstand sich selbst als Versuch, den von den EU-Mitgliedstaaten gebildeten Pfeiler innerhalb der Nördlichen Dimension - eine Initiative Finnlands aus dem Jahre 1997, die im Jahre 2006 in eine gemeinsame Politik Norwegens, Islands, Russlands und der Europäischen Union überführt wurde $-\mathrm{zu}$ stärken. ${ }^{15}$ Kurz darauf erteilte der Europäische Rat der Kommission ein Mandat zur Ausarbeitung der Ostseestrategie. Die Kommission schwächte in der Folgezeit die auf Russland, Norwegen und Island gerichteten Aspekte der Strategie ab und formte sie in eine interne Strategie der Europäischen Union um. Von August 2008 bis Februar 2009 führte die Kommission dazu schließlich eine öffentliche Konsultation mit den verschiedenen Interessengruppen in der Region durch. ${ }^{16}$ Der auf dieser Grundlage erarbeitete Aktionsplan griff die Empfehlungen im Wesentlichen auf. ${ }^{17}$ Dieses partizipatorische Instrument wird nunmehr im Rahmen von jährlich stattfindenden Foren zur Ostseestrategie fortgeführt, die eine Plattform zur Vernetzung, Diskussion und zum Meinungsaustausch über die Strategie und

12 Wilfried Görmar: Makroregionale Strategien: eine neue Dimension europäischer Zusammenarbeit?, in: Informationen zur Raumentwicklung 8/2010, S. 577-589, hier S. 582.

13 Vgl. Wilfried von Bredow: Antagonistische Kooperation als Form der Systemkonkurrenz, in: Manfred Funke (Hrsg.): Friedensforschung - Entscheidungshilfe gegen Gewalt, München 1975, S. 313-320.

14 Vgl. Christopher Beazley: A EU-Strategy for the Baltic Sea Region: Making a Success of the 2004 Enlargement, in: Baltic Rim Economies 6/2007, S. 14.

15 Die Nördliche Dimension war auf finnische Initiative hin im Jahre 1999 zu einer Politik der Europäischen Union entwickelt worden, die den besonderen Bedingungen der nördlichen Mitgliedstaaten in ihrer engen Nachbarschaft zu Russland Rechnung tragen sollte. Auf dem Gipfel in Helsinki im November 2006 wurde die Nördliche Dimension schließlich in eine gemeinsame Politik der vier Partner Europäische Union, Russland, Norwegen und Island überführt mit dem Ziel, einen gemeinsamen Rahmen für Dialog und konkrete Zusammenarbeit, die Stärkung von Stabilität, Wohlstand und wirtschaftlicher Zusammenarbeit zu schaffen.

16 Vgl. Rikard Bengtsson: An EU Strategy for the Baltic Sea Region: Good Intentions Meet Complex Challenges, in: Swedish Institute for European Policy Studies: European Policy Analysis 9/2009, S. 3; Tom Rostoks: In Place of an Introduction: Why the Baltic Sea Strategy?, in: Žaneta Ozoliņa/Iveta Reinholde/Tom Rostoks (Hrsg.): The EU Strategy for the Baltic Sea Region: A Year After and Beyond, Riga 2010, S. 9-40, hier S. 15.

17 Siehe hierzu die Auswertung von Carsten Schymik/Peer Krumrey: EU Strategy for the Baltic Sea Region. Core Europe in the Northern Periphery?, Stiftung Wissenschaft und Politik: SWP Working Paper FG 1, 08/2009. 
deren Umsetzung bereitstellen, auf denen politische, wirtschaftliche und zivilgesellschaftliche Akteure und Entscheidungsträger wie auch Interessengruppen zusammentreffen. ${ }^{18}$ Die Vernachlässigung der Nicht-EU-Mitglieder, insbesondere Russlands, hat gerade in dieser Phase der Strategieformulierung zu Verstimmungen geführt und wurde schließlich erst in der Folgezeit wieder korrigiert. Allerdings stellen die Auswirkungen der sogenannten Ukraine-Krise auf die EU-Russland-Beziehungen seit 2014 auch die Grundlagen der Zusammenarbeit im Ostseeraum zunehmend infrage. So wurde erstmals in der Geschichte der ,Ostseegipfeltreffen` ein für Anfang Juni 2014 in Turku geplantes Treffen der Mitglieder des Ostseerates abgesagt. ${ }^{19}$

\section{Aufbau und Struktur der Gouvernanz-Architektur im Rahmen der EU-Strategie für den Ostseeraum}

Im Oktober 2009 nahm der Europäische Rat die Ostseestrategie unter der Maßgabe an, dass ihre Umsetzung weder neue Institutionen noch Rechts- und Finanzvorschriften erforderlich macht. Die Strategie setzte sich zum Ziel, (1) den Umweltzustand der Ostsee zu verbessern, (2) eine ausgewogenere Wirtschaftsentwicklung in der Region zu unterstützen, (3) die Region sowohl zugänglicher und attraktiver als auch (4) sicherer zu machen. Diese Bereiche wurden in 15 verschiedene Schwerpunktbereiche mit einer Reihe von Vorzeigeprojekten unterteilt. Der Aktionsplan ist dabei als ,rollender Aktionsplan“ konzipiert worden, das heißt er konnte und sollte rasch bearbeitet und weiterentwickelt werden. Im Ergebnis haben diese Aktualisierungen bereits zu einer Reduzierung der ursprünglich vier Pfeiler der Strategie auf drei Ziele geführt, die den Schutz des Meeres, die Verbesserung der regionalen Infrastruktur und die Steigerung des Wohlstands umfassen. ${ }^{20}$

Nach einem Zwischenbericht zum Stand der Umsetzung der Ostseestrategie im Jahr 2010 wurde der erste umfassende Bericht im Juni 2011 erstellt. Die Kommission hält darin fest, dass die Ostseestrategie zu konkreten Maßnahmen mit einer strafferen Nutzung von Ressourcen, neuen Arbeitsmethoden und Netzwerken geführt habe, es aber weiterer Anstrengungen bedürfe. ${ }^{21}$ Obschon die Strategie zu einem Zeitpunkt ins Leben gerufen wurde als schon viele Mittel der EU-Regionalpolitik aus der Förderperiode 2007-13 gebunden waren, kam es dennoch zu einer Reihe von neuen Projekten - wie zum Beispiel dem ,Baltic Deal', ein Projekt, das mit den Landwirten der ganzen Region zusammenarbeitet, um den Nährstoffeintrag und die damit verbundene Eutrophierung des Meeres zu verringern. Es wird oft als ein Modell für eine verbesserte Verknüpfung verschiedener Politikbereiche - hier Landwirtschaft und Meeresumwelt - dargestellt.

Schließlich führte die Europäische Kommission im Jahr 2013 eine Evaluierung durch und stützte sich dabei auf eine umfangreiche Befragung von über 100 wichtigen Interessengruppen sowie eine unabhängige Einschätzung von externen Experten. Die Bewertung

18 Das erste Jahresforum wurde 2010 in Tallinn abgehalten; es folgten weitere Treffen in Danzig (2011), Kopenhagen (2012), Vilnius (2013) und Turku (2014).

19 Siehe Andrey Makarychev: The Baltic Sea Region: Does Russia fit in?, abrufbar unter: http://www.ponarseur asia.org/ru/node/7018 (letzter Zugriff: 1.10.2014).

20 Da zugleich die Zahl der Schwerpunktbereiche von 15 auf 17 angehoben wurde, ist es jedoch tatsächlich fraglich, ob die Strategie damit in der Praxis auch gestrafft worden ist. Immerhin sind die horizontalen Maßnahmen von 13 auf fünf verringert worden. Siehe hierzu auch Tobias Etzold: The EU Strategy for the Baltic Sea Region: Linking the Regional with the EU Level, in: Government Gazette, Baltic Sea Special, March 2013, S. 11.

21 Europäische Kommission: Mitteilung der Europäischen Kommission über die Strategie für den Ostseeraum, 2012, S. 4. 
gelangte zu dem Schluss, dass „, [k]lare Ergebnisse in Form von Projekten und einer integrierteren politischen Entscheidungsfindung [...] bereits ersichtlich [seien], auch wenn bei der Umsetzung und Planung weitere Verbesserungen notwendig [seien]."22 Gleichzeitig wurde auch eine Reihe von Problemen identifiziert, insbesondere der Mangel an politischem Willen und Führung in vielen Mitgliedstaaten in der Makroregion. Obwohl das Fehlen nationaler Ressourcen sicherlich mit dem politischen Desinteresse einiger Regierungen erklärt werden kann, so hat sicherlich auch die Komplexität der Gouvernanz-Architektur der Ostseestrategie einen Teil dazu beigetragen, dass Mitgliedstaaten und Partnerländer der Europäischen Union gleichermaßen der Strategie immer noch mit Skepsis begegnen.

Nach den mit dem Aktionsplan vom Februar 2013 vorgelegten Überarbeitungen verfolgt die Ostseestrategie nunmehr die drei Hauptziele Umweltschutz, wirtschaftliche Entwicklung und Verbesserung der Infrastruktur. Diesen Zielen sind insgesamt 17 Schwerpunktbereiche - beispielsweise wie etwa ,Bio-Diversität' und ,Innovation' sowie fünf horizontale Aktionen, beispielsweise ,Raumordnung' und ,Nachbarn“ - zugeordnet. Für die Schwerpunktbereiche sind jeweils zwei bis drei Koordinatoren aus unterschiedlichen Mitgliedstaaten verantwortlich. Organisationen wie der Ostseerat, die Helsinki-Kommission (HELCOM beziehungsweise Baltic Marine Environment Protection Commission) und ,Visionen und Strategien für die Ostseeregion' (Visions and Strategies around the Baltic Sea, VASAB) spielen als Koordinatoren ebenso eine aktive Rolle wie auch bei der Umsetzung der Ostseestrategie selbst. Die Koordinatoren dieser Schwerpunktbereiche sind in der Regel Mitarbeiter nationaler oder subnationaler Behörden sowie internationaler Organisationen. Gemeinsam mit den Leitern der horizontalen Aktionen spielen sie eine tragende Rolle bei der Umsetzung der Strategie, da sie die Akteure ihrer jeweiligen Bereiche politikfeld- und länderübergreifend zusammenführen. Ähnlich wie bereits im Falle der EUDonaustrategie geschehen, sind oder werden nunmehr auch im Falle der Ostseestrategie sogenannte Lenkungsausschüsse eingerichtet, die interessierte und kompetente Vertreter aus nationalen Einrichtungen in beratender Funktion zusammenbringen und den Koordinator in seiner Arbeit unterstützen sollen. In übergeordneter Funktion wird die Umsetzung auf nationaler - und im Falle der drei Bundesländer auch auf regionaler - Ebene von sogenannten nationalen Kontaktpunkten unterstützt, die die Umsetzung und Koordination der Strategie auf dieser Ebene insgesamt betreuen. Aufgrund der Unterschiede im Bereich der nationalen Verwaltungen haben sich hier im Wirkungsbereich der Ostseestrategie sehr unterschiedliche Praktiken entwickelt. In jedem Fall lässt sich aber festhalten, dass der Erfolg bei der Umsetzung der Ziele der EU-Ostseestrategie maßgeblich vom politischen Willen der beteiligten Akteure in den einzelnen Mitgliedstaaten und -regionen abhängig ist. Zugleich nimmt die Kommission eine wichtige Position bei der Steuerung und dem Monitoring der Strategie ein. Insbesondere spielt sie bei der Beratung einzelner Mitgliedstaaten und Koordinatoren wie auch bei der Überarbeitung der Aktionspläne eine bedeutende Rolle und wird hierbei von der hochrangigen Gruppe im EU-Ministerrat unterstützt, die aus Regierungsvertretern der beteiligten EU-Mitgliedstaaten (und Norwegen) besteht.

Trotz der ,drei Nein' deutet sich gerade im Bereich der institutionellen Architektur an, dass sich mit der Schaffung der Lenkungsausschüsse, Schwerpunktbereichsleiter und nationalen Kontaktstellen ein politikfeld- und raumbezogenes transgouvernementales Netzwerk konstituiert. Eine Besonderheit besteht dabei darin, dass der Europäischen Kommis-

22 Europäische Kommission: Bericht zum Mehrwert der makroregionalen Strategien, 2013, S. 12. 
sion hierbei eine herausragende Funktion bei Koordination und Agenda-Setting zufällt, wie die Ergebnisse einer 2013 durchgeführten Untersuchung unter den Schwerpunktbereichsleitern der Ostsee- und Donaustrategie gezeigt haben. ${ }^{23}$

\section{Die Wirkung der EU-Ostseestrategie auf die Ostseezusammenarbeit}

\section{Die horizontale Interaktion zwischen regionalen Organisationen und internationalen Konventionen im Ostseeraum}

Die Ostseestrategie greift - wie andere makroregionale Strategien der Europäischen Union auch - auf bereits bestehende Institutionen der regionalen Zusammenarbeit zurück. Der Ostseerat und HELCOM sind im Ostseeraum zwei der zentralen zwischenstaatlichen Organisationen. In beiden Gremien ist die Europäische Union sowohl als Institution als auch durch ihre Ostseeanrainerstaaten vertreten. Allerdings war der Einfluss der Europäischen Union - vertreten durch die Europäische Kommission (und im Falle des Ostseerates nunmehr durch den Europäischen Auswärtigen Dienst) - in beiden Organisationen insgesamt relativ gering. Mit der Ostseestrategie ist es der Kommission jedoch gelungen, eine stärker sichtbare und aktivere Rolle einzunehmen. Gleichzeitig hat sich der Ostseerat beim Management einer ganzen Reihe von Schwerpunkten und der Umsetzung von Leuchtturm-Projekten im Rahmen der Ostseestrategie hervorgetan. Darüber hinaus stellt der Ostseerat auch eine gemeinsame Plattform für die regionale Zusammenarbeit mit Russland bereit.

Die im Bereich Umwelt vereinbarten Ziele der Ostseestrategie und ihre Schwerpunktbereiche überlappen sich mit einigen der zentralen Aufgaben von HELCOM, dem Exekutivorgan der Helsinki-Konvention, die im Jahr 1974 abgeschlossen wurde. Die Mitglieder von HELCOM sind die acht EU-Ostseeanrainerstaaten, die Europäische Union und Russland. ${ }^{24}$ Das Ziel von HELCOM besteht im Schutz der Meeresumwelt der Ostsee. Die Helsinki-Konvention, die 1992 verabschiedet wurde, löste das im Jahr 1974 getroffene Abkommen ab und trat schließlich 2000 in Kraft. Seit dem Jahr 2007 bildet der Ostseeaktionsplan der HELCOM die zentrale Arbeitsgrundlage für die Umweltzusammenarbeit in der Makroregion. ${ }^{25}$ HELCOM kann nur Empfehlungen aussprechen, aber keine den Mitgliedern gegenüber rechtlich verbindlichen Beschlüsse oder Richtlinien verabschieden.

Während die Ostseestrategie den Regionalorganisationen nunmehr die Möglichkeit einräumt, ihre jeweiligen Aktivitäten in einen weiteren Kooperationsrahmen einzubetten und stärker auf andere Kooperationspartner abzustimmen, kann die Europäische Union wiederum von der Expertise und Kompetenz der jeweiligen Akteure profitieren. Diese Art ,sanfter' Koordinierung im politischen Raum der Ostseekooperation ist für alle beteiligten Institutionen vorteilhaft, weil sie - in den Worten von Esko Antola - gewissermaßen ,identitätsschonend“ ist. ${ }^{26}$ In diesem Sinne hat auch der EU-Ministerrat die Mitgliedstaaten dazu

23 Stefan Gänzle/Johann-Jakob Wulf: The Emerging Core of the EU's Macro-regional Governance Architecture: Mapping the Roles, Tasks and Self-Perceptions of Priority Area Coordinators and Horizontal Action Leaders in the EU Strategies for the Baltic Sea and the Danube Regions, University of Agder: ISL Working Paper 1/2014; vgl. Stefan Gänzle: Macro-regional Strategies \& the EU. Building Inter \& Trans Government Relations, in: Public Administration Times. International Supplement, 1/2014, S. 14.

24 Norwegen und die Tschechische Republik haben die Konvention ebenfalls unterzeichnet.

25 Vgl. Kristine Kern: Governance for Sustainable Development in the Baltic Sea Region, in: Journal of Baltic Studies 1/2011, S. 67-81.

26 Vgl. Esko Antola: EU Strategy for the Baltic Sea Region. Report for the Konrad-Adenauer-Stiftung London Office, London 2009, S. 11. 
aufgerufen, Synergieeffekte zwischen der Ostseestrategie und multilateralen Kooperationsstrukturen und -netzen im Ostseeraum sowie mit internationalen Finanzinstitutionen auszuloten. Durch eine bessere Koordination und wirksamere Nutzung der Kommunikationskanäle und -foren soll die Effizienz der Interventionen in der Makroregion gesteigert werden. ${ }^{27}$

Das Zusammenspiel zwischen der Ostseestrategie und dem HELCOM Ostseeaktionsplan beispielsweise ist recht deutlich, schließlich empfiehlt die Strategie ausdrücklich seine Umsetzung durch die Mitglieder. In einem Arbeitspapier der HELCOM wird dabei auch festgehalten, dass eine wechselseitige Kohärenz zwischen Ostseestrategie und dem Ostseeaktionsplan besteht, „[...] whereby environmental information collected under HELCOM framework will also serve the Strategy, while the updated Strategy will streamline its future activities and projects according to the common targets, thus supporting the existing commitments under HELCOM and the reaching of a good environmental status of marine waters by 2021."28 Ohnehin bilden in vielen Bereichen die von HELCOM bereitgestellten Messdaten - etwa zur Verschmutzung der Ostsee mit Phosphaten - eine Grundlage für die Ostseestrategie. Ferner unterstützt die Strategie den sektorenübergreifenden Ansatz des Ostseeaktionsplans im Bereich des Umweltschutzes und wertet damit den von HELCOM verfolgten Ökosystemansatz auf, der oftmals auf den Widerstand organisierter Interessen stößt (zum Beispiel in der Landwirtschaft).

Während HELCOM in der Lage ist, Entscheidungen der Europäischen Union in Brüssel zu beeinflussen, kann die Europäische Union wiederum HELCOM als eine Art regionaler Umweltschutzbehörde nutzen. Der Fall der Meeresstrategie-Rahmenrichtlinie ${ }^{29}$ zeigt die Auswirkung der makroregionalen Strategien auf das institutionelle Zusammenspiel zwischen HELCOM und der Europäischen Union. Die Meeresstrategie-Rahmenrichtlinie basiert auf den Erfahrungen des Ostseeaktionsplans von HELCOM und die Kommission verwendet den makroregionalen Ansatz, um die Umsetzung der HELCOM-Beschlüsse systematisch zu verbessern. Während HELCOM-Empfehlungen lediglich eine politische Bindewirkung zu entfalten vermögen, müssen die EU-Richtlinien in nationales Recht umgesetzt werden. ${ }^{30}$ Vor diesem Hintergrund ist allerdings nicht auszuschließen, dass einige HELCOM-Mitglieder dem Gremium in Zukunft größerem Widerstand bei der Verabschiedung solcher ,Empfehlungen“ entgegensetzen werden.

\section{Die Einbindung der subnationalen Ebene und der Zivilgesellschaft}

Makroregionale Strategien bieten sowohl subnationalen Gebietskörperschaften als auch zivilgesellschaftlichen Akteuren neue Betätigungsfelder. Durch ihren Zusammenschluss in transnationalen Netzwerken können sie sich zu konstitutiven Elementen der Makroregionen entwickeln. In der Ostsee-Region spielen die mehr als 100 Mitgliedstädte umfassende Union der Ostseestädte (Union of the Baltic Cities) sowie das baltische Metropolen-Netz-

27 Rat der Europäischen Union: Mitteilung an die Presse. 3152. Sitzung des Rates. Allgemeine Angelegenheiten. Brüssel, den 15. November 2011, Dok. 16836/11, S. 12.

28 HELCOM: Baltic Marine Environment Protection Commission 34th Meeting Helsinki, Finland, 5-6 March 2013, S. 1.

29 Richtlinie 2008/56/EG des Europäischen Parlaments und des Rates vom 17. Juni 2008 zur Schaffung eines Ordnungsrahmens für Maßnahmen der Gemeinschaft im Bereich der Meeresumwelt (Meeresstrategie-Rahmenrichtlinie), in: Amtsblatt der EU, Nr. L 164 vom 25. Juni 2008, S. 19-40.

30 Vgl. Judith van Leeuwen/Kristine Kern: The External Dimension of EU Marine Governance: Institutional Interplay between the European Union and the International Maritime Organisation, in: Global Environmental Politics 1/2013, S. 69-87. 
werk (Baltic Metropoles Network) bei der Umsetzung der Ostseestrategie eine aktive Rolle. Gerade die Zusammenarbeit zwischen den Hansestädten und auch die Partnerschaftsbeziehungen zwischen vielen dieser Städte bilden ein Substrat, das einen positiven Einfluss auf die Umsetzung von makroregionalen Strategien hat. In einigen Schwerpunktbereichen fungieren subnationale Akteure auch als Koordinatoren; so etwa Mecklenburg-Vorpommern im Schwerpunktbereich Tourismus.

Trotz dieser positiven Entwicklungen bestehen auch Defizite bei der Einbindung von lokalen und regionalen Gebietskörperschaften in die Ostseestrategie. So zeigt eine OnlineUmfrage unter den Schwerpunktbereichskoordinatoren der Ostsee, dass nur sehr wenige Städte in Lenkungsgruppen vertreten sind. ${ }^{31}$ Dennoch bereitet die Ostseestrategie den Boden für eine Transnationalisierung der Zivilgesellschaft und der subnationalen Gebietskörperschaften im Ostseeraum. Da makroregionale Gouvernanz nicht auf Nationalstaaten allein beschränkt ist, macht sie dauerhaft die Institutionalisierung neuer Formen der Zusammenarbeit erforderlich. Transnationale Institutionen werden so zu einem konstitutiven Element der Makroregionen, einschließlich hybrider Arrangements von staatlichen und nichtstaatlichen Akteuren. ${ }^{32}$ Insgesamt lassen sich dabei drei Arten der Transnationalisierung unterscheiden: (1) die Transnationalisierung bestehender internationaler und zwischenstaatlicher Organisationen (zum Beispiel HELCOM), die Nichtregierungsorganisationen und subnationalen Akteuren zunehmend Zugang zu diesen Institutionen verschafft; (2) die transnationale Vernetzung bestehender nationaler Institutionen - wie die ,Coalition Clean Baltic'; und schließlich (3) die Schaffung neuer transnationaler Institutionen, die auf einem Multi-Stakeholder-Ansatz basieren und die Beteiligung der Zivilgesellschaft fördern. ${ }^{33}$ Die Kombination dieser drei Formen bietet Optionen für eine unmittelbare Einbindung sowohl der subnationalen Gebietskörperschaften als auch der Zivilgesellschaft auf makroregionaler Ebene. Diese Entwicklung eröffnet somit neue Möglichkeiten, aber sie führt auch zu neuen Herausforderungen, weil die Beteiligung der Akteure in Makroregionen grundsätzlich auch Fragen der Legitimität aufwirft. Immerhin zielen gerade einige der Projekte aus der horizontalen Maßnahme ,INVOLVE‘ darauf ab, pan-baltische Organisationen aus Zivilgesellschaft, Wirtschaft und Wissenschaft in die Umsetzung der Ostseestrategie einzubeziehen und Experten aus Nichtregierungsorganisationen, insbesondere dem Ostsee NGO Network, bei der Vorbereitung und Durchführung von Maßnahmen der Ostseestrategie zu beteiligen. Dies erfordert aber auch den Aufbau von Kapazitäten, die es den Mitgliedern dieses Netzwerkes ermöglichen, grenzüberschreitend zusammenarbeiten.

\section{Die Einbindung von Nicht-EU-Mitgliedern am Beispiel Russlands}

Da die Ostseestrategie auf Aktivitäten verweist, die im beiderseitigen Interesse der EUMitgliedstaaten und ihrer Nachbarländer liegen, ist in vielen Bereichen der Strategie eine enge Zusammenarbeit mit Drittländern, insbesondere Russland, erforderlich und sinnvoll.

31 Vgl. Gänzle/ Wulf: The Emerging Core of the EU's Macro-regional Governance, 2014.

32 Vgl. Marko Joas/Kristine Kern/Siv Sandberg: Actors and Arenas in Hybrid Networks Implications for Environmental Policy-making in the Baltic Sea Region, in: Ambio: A Journal of the Human Environment 3/2007, S. 237-242.

33 Vgl. Kristine Kern/Tina Löffelsend: Governance beyond the Nation State. Transnationalization and Europeanization of the Baltic Sea Region, in: Marko Joas/Detlef Jahn/Kristine Kern (Hrsg.): Governing a Common Sea. Environmental Policies in the Baltic Sea Region, London, 2008, S. 115-141; Kern: Governance for Sustainable Development, 2011. 
Dies gilt insbesondere im Bereich der maritimen Überwachung. ${ }^{34}$ Bestehende regionale Organisationen wie etwa HELCOM, VASAB und Ostseerat bieten daher eine geeignete Grundlage für die Zusammenarbeit zwischen den EU-Mitgliedstaaten und Nicht-EU-Ländern. Obwohl die Ostseestrategie stärker auf die EU-Mitgliedstaaten gerichtet ist, gibt es auch mit Blick auf Russland und Norwegen Kooperationsangebote. Dabei verweisen viele Maßnahmen auch auf die Nördliche Dimension, einer Kooperation zwischen Europäischer Union, Russland, Norwegen und Island. ${ }^{35}$ In diesem Sinne äußerte sich auch der Generaldirektor des Ostseerats: „The Strategy has improved transparency in regional cooperation, and the [Council of the Baltic Sea States] is together with e.g. HELCOM and the Northern Dimension one of several platforms on which [European Union Strategy for the Baltic Sea Region] cooperation can occur, with participation also by non-EU[-Baltic Sea Region] countries. " ${ }^{\text {36 }}$ Mit der Ostseestrategie hat die Europäische Union einen Bezugsrahmen für die Akteure und Institutionen regionaler Zusammenarbeit im Ostseeraum geschaffen. Heute werden viele Aktionen und Projekte im Bereich von, Rettung der Ostsee ' mittels der Umweltpartnerschaft der Nördlichen Dimension, HELCOM, dem Ostseerat, sowie neuen Initiativen für den Süd-Ost-Ostseeraum umgesetzt. In der Tat scheint die Ostseestrategie als Bezugspunkt für die Zusammenarbeit im Ostseeraum auch für Nicht-EU-Mitglieder, wie insbesondere Russland, prinzipiell akzeptabel zu sein.

Der Einschluss von - beziehungsweise die grundsätzliche Offenheit gegenüber - NichtEU-Mitgliedern ist ein Element, das allen makroregionalen Strategien bislang gemeinsam ist. Dabei hat sich in der Anfangsphase der Ostseestrategie gerade Russland sehr skeptisch gezeigt, da es sich als nicht ausreichend und gleichberechtigt in die Beratungen zur Ostseestrategie eingebunden erachtete. Zumindest für Nordwest-Russland schuf die russische Regierung mit der Formulierung einer eigenständigen Nordwest-Strategie einen Rahmen, um an die Ostseestrategie der Europäischen Union anknüpfen zu können. ${ }^{37}$ In der russischen Regionalstrategie findet sich eine Reihe von Projekten, die an laufende Maßnahmen der Ostseestrategie anschlussfähig sind. Dies gilt insbesondere für den Bereich der wirtschaftlichen und sozialen Entwicklung in der Nordwest-Strategie. Außerdem ist eine ganze Reihe von Projektanträgen mit russischer Beteiligung bei der zweiten Ausschreibungsrunde der , Seed Money Facility ‘ im Rahmen der Ostseestrategie erfolgreich gewesen.

Es ist davon auszugehen, dass makroregionale Zusammenarbeit, insbesondere im Hinblick auf die Gouvernanz-Architektur, Möglichkeiten zum Wissensaustausch und -transfer sowie zur Sozialisierung der beteiligten Akteure eröffnet. Im Gegensatz zu anderen Regionen an der russischen Außengrenze hat sich die Zusammenarbeit des Landes mit westlichen Partnern gerade im Ostseeraum stärker normalisiert wie auch , subnationalisiert ${ }^{\star}$. So ist beispielsweise das Ostseeratssekretariat gemeinsam mit Vertretern des Turku-Prozesses in die Koordination der Maßnahmen unter der horizontalen Maßnahme ,Nachbarn“ auch praktisch eingebunden. Die Ostseestrategie selbst bildete 2010 den unmittelbaren Anlass

34 Vgl. Europäische Kommission: Strategie für den Ostseeraum, 2012, S. 9.

35 Vgl. Europäische Kommission: Commission Staff Working Document Accompanying the Document Report from the Commission to the European Parliament, the Council, the European Economic and Social Committee and the Committee of the Regions Concerning the Added Value of Macro-regions, Brussels, SWD (2013) 233, S. 13.

36 Jan Lundin: The Council of the Baltic Sea States in the Era of Regional Strategies, Networks and Projects, in: Baltic Rim Economies, 28.02.2013, S. 15.

37 Siehe Russian Federation: Strategy of Social and Economic Development of the North-West Federal District until 2020, 2012; Russian Federation: Action Plan on the Strategy of Social and Economic Development of the North-West Federal District until 2020, 16.10.2012, No. 1939-p. 
dafür, den Turku-Prozess ins Leben zu rufen. Dabei handelt es sich um eine Initiative zur Intensivierung der Zusammenarbeit der Städte Turku, St. Petersburg und Hamburg. Der Turku-Prozess zielt vorrangig auf die praktische Zusammenarbeit mit russischen Partnern ab und profitiert von den langjährigen Erfahrungen der drei Partnerstädte. Eine Reihe von Expertenkomitees befasst sich unter anderem mit der Förderung der Energieeffizienz und des Energiesparens bei Neubau- und Sanierungsmaßnahmen in allen drei Städten, der wachsenden Mobilität der Arbeitskräfte, dem Umweltschutz sowie der Entwicklung neuer Kooperationsmodelle, die die Kontakte zwischen den Forschungsinstituten, Universitäten und der Wirtschaft verbessern sollen. Ferner hat der Ostseerat auch ein Modernisierungsprogramm für den südöstlichen Ostseeraum (SEBA) verabschiedet, das schwerpunktmäßig auf die Region Kaliningrad abzielt.

Aus diesen bislang positiven Entwicklungen kann grundsätzlich geschlossen werden, dass sich Fragen sicherheitspolitischer Natur, wie sie im Zuge der Ukraine-Krise zwischen der Europäischen Union und Russland in den Vordergrund getreten sind, in funktionalen Bereichen der ,low politics` und unter Einschluss subnationaler Akteure noch eher zurückstellen lassen. Allerdings sind die Auswirkungen der sich im Zuge der Ukraine-Krise verändernden Beziehungen zwischen Russland und der Europäischen Union auch in der Ostseeregion spürbar.

\section{Erfolge und Defizite der Ostseestrategie}

Fünf Jahre nach ihrer Initiierung hat sich die Ostseestrategie als ein neuartiges Instrument zur Verbindung der europäischen territorialen Zusammenarbeit mit einer Reihe politikfeldübergreifender Ziele etabliert. Wie sieht die Bilanz insgesamt aus?

Obwohl die Ostseestrategie einen Beitrag zu leisten versucht, die Vielzahl von Aktivitäten unterschiedlicher Akteure im Ostseeraum besser aufeinander zu beziehen und mithin behutsam zu straffen, kann dies nicht ohne eine Erhöhung der durch diese zusätzliche Koordination entstehenden Transaktionskosten geschehen. Zwar hat sich bereits jetzt die Gouvernanz-Architektur kontinuierlich weiterentwickelt und bietet eine gemeinsame Basis für die Zusammenarbeit und die Umsetzung der Strategie. Dennoch führt die geschaffene Gouvernanz-Architektur bei einigen der beteiligten Akteure angesichts ihrer begrenzten finanziellen und personellen Ressourcen zu erheblichen Problemen.

Die makroregionale Gouvernanz-Architektur verbindet EU-Mitgliedstaaten und Partnerländer, internationale Organisationen, subnationale Behörden und private Akteure durch nationale Kontaktstellen, Schwerpunktbereichs-Koordinatoren, Leuchtturmprojektleiter und Stellen, die für die Umsetzung von Programmen/Finanzinstrumenten verantwortlich sind. Auf der Grundlage sektoraler Interdependenz und transgouvernementaler Netzwerke, bietet das System der Koordinatoren einen „Schlüssel für den künftigen Erfolg“. ${ }^{38}$ Das System, jeweils zwei Schwerpunktbereichskoordinatoren einzusetzen, hat beispielsweise im Bereich ,Energie‘ eine Intensivierung der Zusammenarbeit zwischen Lettland und Dänemark angestoßen. Jedoch bleibt es eine wichtige Aufgabe sicherzustellen, dass diese Koordinatoren durch entschlossene und engagierte Lenkungsausschüsse unterstützt werden, die die Reichweite der Strategie weit über die inneren Kreise des zuständigen Ministeriums hinaustragen. Sollten sich die in der Ostseestrategie vorgesehenen Gou-

38 Interview des Autors mit einem Mitglied der Ständigen Vertretung Schwedens, 2.7.2013. 
vernanz-Strukturen mittel- und langfristig bewähren, dann wird damit ein innovativer politischer Raum zur Lösung praktischer Herausforderungen im Ostseeraum geschaffen.

Die Ostseestrategie hat die Koordinierung der bestehenden Organisationen, Netzwerke, Projekte und Finanzierungsinstrumente und die Zusammenarbeit zwischen den Akteuren in der Makroregion, einschließlich des Privatsektors, wohl verbessert; sie hat zu neuen Projekten geführt, die beispielsweise darauf abzielen, die Eutrophierung der Ostsee zu reduzieren oder die bestehende Verkehrsinfrastruktur zu verbessern. Die nachhaltige Einbindung von nichtstaatlichen Akteuren bleibt aber eine wichtige Herausforderung. Nach einem holprigen Start hat die Strategie zu einer stärkeren Beteiligung der russischen Partner in Bereichen wie Umweltschutz, Wasserqualität und Innovation geführt, insbesondere bei den subnationalen Akteuren in Nordwest-Russland. Eine wichtige Frage bleibt, ob die Nördliche Dimension oder der Ostseerat als eine zentrale Plattform für diese Interaktion dienen kann.

Die Vielzahl der 100 Leuchtturmprojekte - wie die Projekte ,Cleanship“ und BALTFISH (Baltic Sea Fisheries Forum), die auf eine Verringerung der Meeresverschmutzung durch Schiffe und eine Verbesserung der Zusammenarbeit im Fischereimanagement abzielen - waren bereits vor der Verabschiedung der Ostseestrategie beschlossen worden. Sie haben aber sicherlich von der verstärkten Aufmerksamkeit im Zuge der Ostseestrategie profitieren können. Es gibt auch einige Hinweise, dass Spin-off-Projekte eingerichtet und Projektideen von anderen Akteuren wie zum Beispiel nationalen Regierungen aufgegriffen werden. ${ }^{39}$ Der Gesamterfolg der Strategie wird in den kommenden Jahren weitgehend davon abhängen, ob es gelingen wird, die vorhandenen knappen Ressourcen besser zu bündeln und effizienter zu nutzen.

Die Ostseestrategie wirkt unmittelbar auf bestehende Institutionen und internationale Konventionen wie HELCOM und den Ostseeaktionsplan ein. Umgekehrt beeinflussen die HELCOM-Richtlinien EU-Entscheidungsprozesse in Brüssel und machen die EU-Rechtsvorschriften auf der Grundlage dieser Richtlinien für alle Mitgliedstaaten der HELCOM verbindlich. Darüber hinaus verbessert die Strategie die Umsetzung bestehender EURechtsvorschriften, zum Beispiel REACH ${ }^{40}$ die Bestimmungen zu den Europäischen Verkehrsnetzen (TEN-T), die Wasserrahmen-Richtlinie ${ }^{41}$ und die Meeresstrategie-Rahmenrichtlinie. Die EU-Kommission wird auch weiterhin eine Schlüsselrolle in der Ostsee-Makroregion spielen, aber ihre Rolle muss durch die politische Führung der Mitgliedstaaten in der Region selbst ergänzt werden. Viele der beteiligten Länder - EU-Mitgliedstaaten und Nicht-EU-Mitglieder gleichermaßen - müssen ein stärkeres Engagement und mehr Eigenverantwortung entwickeln.

39 Vgl. Europäische Kommission: Bericht zum Mehrwert der makroregionalen Strategien, 2013, S. 4.

40 Verordnung (EG) Nr. 1907/2006 des Europäischen Parlaments und des Rates vom 18. Dezember 2006 zur Registrierung, Bewertung, Zulassung und Beschränkung chemischer Stoffe (REACH), zur Schaffung einer Europäischen Chemikalienagentur, zur Änderung der Richtlinie 1999/45/EG und zur Aufhebung der Verordnung (EWG) Nr. 793/93 des Rates, der Verordnung (EG) Nr. 1488/94 der Kommission, der Richtlinie 76/769/EWG des Rates sowie der Richtlinien 91/155/EWG, 93/67/EWG, 93/105/EG und 2000/21/EG der Kommission, in: Amtsblatt der EU, Nr. L 396 vom 30. Dezember 2006, S. 1-851.

41 Richtlinie 2000/60/EG des Europäischen Parlaments und des Rates vom 23. Oktober 2000 zur Schaffung eines Ordnungsrahmens für Maßnahmen der Gemeinschaft im Bereich der Wasserpolitik, in: Amtsblatt der EU, Nr. L 327 vom 22. Dezember 2000, S. 1-73. 